\title{
Equations-of-motion formulation of many-body perturbation theory
}

\author{
Esper Dalgaard and Jack Simons $\dagger$ \\ Department of Chemistry, Aarhus University, DK-8000 Aarhus C, Denmark
}

Received 1 March 1977

\begin{abstract}
A formulation of many-body perturbation theory starting from the operator equation $\left[H, Q^{+}\right]=\omega Q^{+}$is presented. The method of solution is based on an operator scalar product, $(X \mid Y)=\operatorname{Tr}\left(X^{+} Y\right)$, which allows us to use resolvent and partitioning techniques to establish Rayleigh-Schrödinger or Brillouin-Wigner perturbation theory for the excitation energy and excitation operator, $Q^{+}$. The excitation operator contains all information about the two states involved in the transition. Specific results are given for removal or addition of an electron and for excitations of particle-hole type and comparison with the Green's function methods is made.
\end{abstract}

\section{Introduction}

It has recently been pointed out by one of us (Simons 1977) that the solutions to operator-level equations may have certain advantages over solutions to the corresponding function-level equations. For a given Hamiltonian $H$, the eigenoperators $Q^{+}$and eigenenergies $\omega$ which obey $\left[H, Q^{+}\right]=\omega Q^{+}$are uniquely determined, apart from multiplication by any operator that commutes with $H$, whereas the solutions $\tilde{Q}^{+}$and $\tilde{\omega}$ of the function-level equation $H \tilde{Q}^{+}|\psi\rangle-\tilde{Q}^{+} H|\psi\rangle=\tilde{\omega} \tilde{Q}^{+}|\psi\rangle$, for a chosen reference function $|\psi\rangle$, are not uniquely determined. That is, for a given $H$ and a given $|\psi\rangle$, which may even be an exact eigenstate of $H$, there are an infinite number of excitation operators $\tilde{Q}^{+}$corresponding to any resulting eigenenergy $\tilde{\omega}$. These eigenenergies $\tilde{\omega}$ may be equal to exact excitation energies or ionisation energies, but the fact that the operators $\tilde{Q}^{+}$are not uniquely determined is a bothersome feature of the solution.

The possibility of formulating quantum mechanics entirely in terms of operators and mappings of operators, i.e. superoperators, was pointed out by Crawford (1958). Similar concepts were employed by Primas (1961) in an operator treatment of manybody perturbation theory. Dirac (1966) found the use of operator equations instead of Schrödinger equations to be almost a necessity in quantum electrodynamics. A particularly compact operator formulation of resolvent and partitioning techniques in perturbation theory has been given by Löwdin (1968).

In the present paper emphasis will be placed on how one can solve operatorlevel equations of motion using perturbation theory and how the resulting formalism is applied in a straightforward manner to treat, as examples, electronic excitation

$\uparrow$ Alfred P Sloan Fellow 1974-1978, Camille and Henry Dreyfus Fellow. On leave from the Chemistry Department, University of Utah, Salt Lake City, Utah 84112. USA. 
and ionisation processes in atoms and molecules. In $\$ 2$ the derivation of our working equations is achieved through the introduction of an operator scalar product which fulfils all of the mathematical requirements and, hence, permits us to use the concepts of projection onto a particular operator and its orthogonal complement. General expressions are given for the $n$ th-order excitation energies and excitation operators in terms of the perturbation operator $V$ and a specific zeroth-order reference operator. In $\$ 3$ we demonstrate how this perturbative treatment can be applied to problems of electronic ionisation which, in zeroth order, are described by the addition (or removal) of a single electron to (or from) a spin orbital. Specific results are given for the electron affinities (or ionisation potentials) through third order and comparison with results from Green's function theory and Rayleigh-Schrödinger perturbation theory is made. Section 4 contains an analogous treatment and discussion of electronic excitations which, in zeroth order, are described as promotion of a single electron from an occupied spin orbital to an unoccupied orbital. Our concluding remarks together with a review of the results are given in $\$ 5$.

\section{General formalism}

We consider the equation of motion for an excitation operator, $Q^{+}$, in the form

$$
\left[H, Q^{+}\right]=\omega Q^{+} \text {. }
$$

It is natural to expect that there will be solutions to equation (1) which are power series in a perturbation parameter, i.e.

$$
\begin{aligned}
& Q^{+}=Q_{0}^{+}+Q_{1}^{+}+Q_{2}^{+}+\ldots \\
& \omega=\omega_{0}+\omega_{1}+\omega_{2}+\ldots
\end{aligned}
$$

when the Hamiltonian is a sum of a zeroth-order term and a perturbation:

$$
H=H_{0}+V \text {. }
$$

Introducing equations (2) and (3) in (1) and collecting terms of the same order in $V$, we find a set of Rayleigh-Schrödinger perturbation theory equations

$$
\begin{aligned}
& \left(\hat{H}_{0}-\omega_{0}\right) Q_{0}^{+}=0 \\
& \left(\hat{H}_{0}-\omega_{0}\right) Q_{1}^{+}=\left(\omega_{1}-\hat{V}\right) Q_{0}^{+} \\
& \left(\hat{H}_{0}-\omega_{0}\right) Q_{2}^{+}=\omega_{2} Q_{0}^{+}+\left(\omega_{1}-\hat{V}\right) Q_{1}^{+}
\end{aligned}
$$

The superoperator notation $\hat{H}_{0} X=\left[H_{0}, X\right]$ and $\hat{V} X=[V, X]$ for any operator $X$, is used (Goscinski and Lukman 1970).

The methods which one might use in order to solve these equations are completely analogous to the treatments of the same equations in ordinary perturbation theory for state vectors. However, both the resolvent and the expansion techniques are based on the existence of a scalar product defined on the particular state-vector space. Thus, we need a similar concept here. The operator scalar product employed by Goscinski and Lukman (1970) in propagator theory will not serve our purpose, because we want to avoid any reference to a particular state, and because it does 
not provide any means of deciding whether two operators are identical or not (i.e. $(X-Y \mid X-Y)=0$ is not necessary and sufficient for $X=Y)$. Instead our development will be based on the following definition of the scalar product, $(X \mid Y)$, between two operators $X$ and $Y$ (Crawford 1958, Primas 1961, Banwell and Primas 1963, Löwdin 1976)

$$
(X \mid Y)=\operatorname{Tr}\left(X^{+} Y\right) .
$$

It is readily checked that this scalar product fulfils the mathematical axioms of a scalar product, namely

$$
\begin{aligned}
& \left(X \mid Y_{1} c_{1}+Y_{2} c_{2}\right)=\left(X \mid Y_{1}\right) c_{1}+\left(X \mid Y_{2}\right) c_{2} \\
& (X \mid Y)=(Y \mid X)^{*} \\
& (X \mid X) \geqslant 0
\end{aligned}
$$

and

$$
(X \mid X)=0 \Longleftrightarrow X=0 .
$$

It is equation (11) which permits us to test whether two operators are equal. A solution of the equations (4)-(6) may now be established in the normal way. Assume that the unperturbed excitation energy, $\omega_{0}$, and excitation operator, $Q_{0}^{+}$, are known and that $\omega_{0}$ is non-degenerate. Equation (5) implies that

$$
\left(Q_{0}^{+}\left|\hat{H}_{0}-\omega_{0}\right| Q_{1}^{+}\right)=\left(Q_{0}^{+}\left|\omega_{1}-\hat{V}\right| Q_{0}^{+}\right) .
$$

Since

$\left(Q_{0}^{+}\left|\hat{H}_{0}\right| Q_{1}^{+}\right)=\operatorname{Tr}\left(Q_{0}\left[H_{0}, Q_{1}^{+}\right]\right)=-\operatorname{Tr}\left(Q_{1}^{+}\left[H_{0}, Q_{0}\right]\right)=\omega_{0}\left(Q_{0}^{+} \mid Q_{1}^{+}\right)$

we see that the first-order correction to the excitation energy is

$$
\omega_{1}=\left(Q_{0}^{+}|\hat{V}| Q_{0}^{+}\right) /\left(Q_{0}^{+} \mid Q_{0}^{+}\right)
$$

In the following we will always assume that

$$
\left(Q_{0}^{+} \mid Q_{0}^{+}\right)=\left(Q_{0}^{+} \mid Q^{+}\right)=1
$$

i.e. we adopt the intermediate normalisation condition. In order to proceed in a manner corresponding to the resolvent technique (Löwdin 1968) we define a superoperator projector, $\hat{\Pi}_{0}$, for the orthogonal complement of $Q_{0}^{+}$. Formally we may write

$$
\hat{\Pi}_{0}=1-Q_{0}^{+}\left(Q_{0}^{+} \mid\right. \text {. }
$$

Two important properties of this operator are

$$
\hat{\Pi}_{0} Q_{0}^{+}=0
$$

and

$$
\left[\hat{H}_{0}, \hat{\Pi}_{0}\right]=0 .
$$

A formal solution of equation (5) for the first-order correction to the excitation operator may now be expressed as

$$
Q_{1}^{+}=\left(\omega_{0}-\hat{H}_{0}\right)^{-1} \hat{\Pi}_{0} \hat{V} Q_{0}^{+}
$$


but in the applications in the following sections it will prove at times more convenient to use an integral representation

$$
Q_{1}^{+}=-\frac{\mathrm{i}}{2} \int_{-\infty}^{\infty} \mathrm{d} t \operatorname{sgn}(t) \exp \left[\mathrm{i}\left(\omega_{0}-\hat{H}_{0}\right) t\right] \hat{\Pi}_{0} \hat{V} Q_{0}^{+} .
$$

The precise definition of the sign function $\operatorname{sgn}(t)$, appearing in this equation will here be chosen to be

$$
\operatorname{sgn}(t)=\lim _{n \rightarrow 0} \begin{cases}-e^{|\eta| t} & \text { when } t<0 \\ e^{-|\eta| t} & \text { when } t \geqslant 0 .\end{cases}
$$

Similarly we find the general expression for the $n$ th-order correction to the excitation energy and excitation operator as

$$
\omega_{n}=\left(Q_{0}^{+-}|\hat{V}| Q_{n-1}^{+}\right)
$$

and

$$
Q_{n}^{+}=\left(\omega_{0}-\hat{H}_{0}\right)^{-1} \hat{\Pi}_{0}\left(\hat{V} Q_{n-1}^{+-1}-\sum_{j=1}^{n-1} \omega_{n-j} Q_{j}^{+}\right)
$$

respectively. Equations (22) and (23) are, of course, identical in form to the results of ordinary, non-degenerate Rayleigh-Schrödinger perturbation theory. A BrillouinWigner form of perturbation theory might similarly be derived, e.g. by means of the partitioning of Löwdin (1968).

In the applications to removal or addition of an electron, and to particle-hole-type excitations, described in the next two sections, the unperturbed excitations will be degenerate when the zeroth-order Hamiltonian is taken to be $H_{0}=\Sigma \epsilon_{r} n_{r}$. Here the number operator for the spin orbital labelled $r$ is denoted $n_{r}$. The degeneracy arises because $H_{0}$ commutes with any function of number operators. Consider e.g. the choice $Q_{0}^{+}=a_{k}^{+} a_{l}$, so that $\hat{H}_{0} Q_{0}^{+}=\left(\epsilon_{k}-Q_{0}^{+}\right) Q_{0}^{+}$. The operator $\tilde{Q}^{+}=a_{k}^{+} a_{l} n_{r} n_{r^{\prime}} \ldots$ obeys the same equation, since

$$
\hat{H}_{0} \tilde{Q}_{0}^{+}=\left(\hat{H}_{0} Q_{0}^{+}\right) n_{r} n_{r^{\prime}} \ldots=\left(\epsilon_{k}-\epsilon_{l}\right) \tilde{Q}_{0}^{+} .
$$

Thus there will be a manifold of operators satisfying equation (4). A complete characterisation of the degeneracy, which arises in this manner, may be achieved by choosing the degenerate operators so as to correspond to excitations from different unperturbed initial states, which can be specified by a set of spin-orbital occupation numbers $f_{r}(s)$. The projectors onto these states can be represented as

$$
P_{s}^{0}=(-1)^{N} \Pi_{r}\left[1-n_{r}-f_{r}(s)\right] \quad N=\sum_{r} f_{r}(s) .
$$

A superoperator, $\hat{P}_{s}^{0}$, is defined by the relation

$$
\hat{P}_{s}^{0} X=X P_{s}^{0}
$$

for any operator $X$. With the operator scalar product of equation (7), $\hat{P}_{s}^{0}$ is Hermitian and

$$
\left(\hat{P}_{s}^{0}\right)^{2}=\hat{P}_{s}^{0}
$$

so that also $\hat{P}_{s}^{0}$ is a projector. When the unperturbed Hamiltonian is taken to be $H_{0}=\Sigma \epsilon_{\mathrm{r}} n_{r}$ we have

$$
\left[\hat{H}_{0}, \hat{P}_{\mathrm{s}}^{0}\right]=0
$$


i.e. $\hat{P}_{s}^{0}$ describes a symmetry of the superoperator $\hat{H}_{0}$. The set of operators $\left\{\hat{P}_{s}^{0}\right\}$ is complete in the sense that

$$
\Sigma \hat{P}_{s}^{0}=\hat{1} \text {. }
$$

We will choose the unperturbed excitation operators to be symmetrised so that

$$
\hat{P}_{s^{\prime}}^{0} Q_{0}^{+}(s)=\delta_{s s^{\prime}} Q_{0}^{+}(s) \text {. }
$$

A few possible representations of the $Q_{0}^{+}(s)$ corresponding to removal of an electron, addition of an electron, and to a particle-hole-type excitation are:

$$
Q_{0}^{+}(s)=\left\{\begin{array}{l}
a_{h} P_{s}^{0} \\
a_{p}^{+} P_{s}^{0} \\
a_{p}^{+} a_{h} P_{s}^{0}
\end{array}\right.
$$

where $p$ and $h$ label orbitals whose occupation numbers are $f_{p}(s)=0$ and $f_{h}(s)=1$, respectively. When evaluating scalar products involving these operators, the following relations are useful

$$
\begin{aligned}
& Q_{0}^{+}(s) Q_{0}^{+}\left(s^{\prime}\right)=0 \\
& Q_{0}(s) Q_{0}^{+}\left(s^{\prime}\right)=\delta_{s s^{\prime}} P_{s}^{0} \\
& Q_{0}^{+}(s) Q_{0}\left(s^{\prime}\right)=\delta_{s s^{\prime}} \times\left\{\begin{array}{l}
a_{h} P_{s}^{0} a_{h}^{+} \\
a_{p}^{+} P_{s}^{0} a_{p} \\
a_{p}^{+} a_{h} P_{s}^{0} a_{h}^{+} a_{p} .
\end{array}\right.
\end{aligned}
$$

In solving the above perturbation equations, (4)-(6), a form of degenerate perturbation theory has to be applied because the unperturbed projectors $\hat{P}_{s}^{0}$ do not commute with $\hat{V}$ and hence the perturbed excitation operators $Q^{+}(s)$ will not obey equation (29). The degeneracy is lifted in first order, however, and the operators given by equation (30) are the correct zero-order combinations of degenerate operators since

$$
\left(Q_{0}^{+}\left(s^{\prime}\right)|\hat{V}| Q_{0}^{+}(s)\right)=\delta_{s s^{\prime}} \omega_{1}(s)
$$

as a consequence of equations (32) and (33). In the following treatment of this degenerate problem, we will keep the normalisation condition as given by equation (15), which now reads

$$
\left(Q_{0}^{+}(s) \mid Q^{+}(s)\right)=1 .
$$

As discussed by, for instance, Condon and Shortley (1935) we may proceed in either of two different ways in order to obtain the perturbative solution for $Q^{+}(s)$. We could redefine $\hat{H}_{0}$ and $\hat{V}$ so as to remove the degeneracy in zero order. A particular way of doing this is to define

$$
\hat{H}_{0}^{1}=\hat{H}_{0}+\sum_{s^{\prime \prime}} Q_{0}^{+}\left(s^{\prime \prime}\right) \omega_{1}\left(s^{\prime \prime}\right)\left(Q_{0}^{+}\left(s^{\prime \prime}\right)\right.
$$

and

$$
\hat{V}^{1}=\hat{V}-\sum_{s^{\prime \prime}} Q_{0}^{+}\left(s^{\prime \prime}\right) \omega_{1}\left(s^{\prime \prime}\right)\left(Q_{0}^{+}\left(s^{\prime \prime}\right)\right)
$$

where $s^{\prime \prime}$ runs over the degenerate manifold, so that the new reference excitation energies, which are of first order in $\hat{V}$ but zero order in $\hat{V}^{1}$, would be

$$
\omega_{0}^{1}(s)=\omega_{0}+\omega_{1}(s) .
$$


The non-degenerate perturbation theory, described above, could then be applied directly.

Another procedure is to solve the Rayleigh-Schrödinger perturbation theory equations successively in the following manner. Assume that the corrections $Q_{1}^{+}(s), \ldots$, $Q_{n-1}^{+}(s)$ have been found. The equation

$$
\left(\hat{H}_{0}-\omega_{0}\right) Q_{n}^{+}(s)=\sum_{j=0}^{n-2} \omega_{n-j}(s) Q_{j}^{+}(s)+\left(\omega_{1}(s)-\hat{V}\right) Q_{n-1}^{+}(s)
$$

has solutions only if the right-hand side is orthogonal to all $Q_{0}^{+}\left(s^{\prime}\right)$, and we assume that this condition is fulfilled. Using the projector onto the complement of the degenerate manifold $\left\{Q_{0}^{+}\left(s^{\prime}\right)\right\}$,

$$
\hat{\Pi}_{0}=\hat{1}-\sum_{s^{\prime \prime}} Q_{0}^{+}\left(s^{\prime \prime}\right)\left(Q_{0}^{+}\left(s^{\prime \prime}\right)\right.
$$

we write $Q_{n}^{+}(s)$ as

$$
Q_{n}^{+}(s)=\hat{\Pi}_{0} Q_{n}^{+}(s)+\sum_{s^{\prime \prime} \neq s} \kappa_{n}\left(s^{\prime \prime}\right) Q_{0}^{+}\left(s^{\prime \prime}\right)
$$

The term for which $s^{\prime \prime}=s$ is excluded because $\left(Q_{0}^{+}(s) \mid Q_{n}^{+}(s)\right)=0$. Only the first part, $\hat{\Pi}_{0} Q_{n}^{+}(s)$, can be determined from equation (39). The coefficient $\kappa_{n}\left(s^{\prime \prime}\right)$ can be derived uniquely from the requirement that the right-hand side of the next higher order equation,

$$
\left(\hat{H}_{0}-\omega_{0}\right) Q_{n+1}^{+}(s)=\sum_{j=0}^{n-1} \omega_{n+1-j}(s) Q_{j}^{+}(s)+\left(\omega_{1}(s)-\hat{V}\right) Q_{n}^{+}(s)
$$

also belongs to the complement of the degenerate manifold. We find, using equation (41) in equation (42), that for $n \geqslant 2$

$\kappa_{n}\left(s^{\prime \prime}\right)=\left(\left(Q_{0}^{+}\left(s^{\prime \prime}\right)\left|\hat{V} \hat{\Pi}_{0}\right| Q_{n}^{+}(s)\right)-\sum_{j=1}^{n-1} \omega_{n+1-j}(s) \kappa_{j}\left(s^{\prime \prime}\right)\right)\left(\omega_{1}(s)-\omega_{1}\left(s^{\prime \prime}\right)\right)^{-1}$

and that $\kappa_{1}\left(s^{\prime \prime}\right)$ vanishes identically.

Thus, the $\hat{\Pi}_{0} Q_{n}^{+}(s)$ component is obtained from equation (23) and the remainder is given in equation (43). The $n$ th-order excitation energy is still given by equation (22).

Transition moments,

$$
M=\left\langle s|R| s^{*}\right\rangle=\operatorname{Tr}\left(|s\rangle\left\langle s|R| s^{*}\right\rangle\langle s|\right)=\operatorname{Tr}\left(P_{s} R Q^{+}(s)\right)
$$

corresponding to the excitation described by the operator $Q^{+}(s)$, as well as the properties of the initial state $|s\rangle$ and the final state $\left|s^{*}\right\rangle$, may also be calculated to any desired order in the perturbation within the present formalism. To this end we need the perturbed projector $P_{s}$, which commutes with the total Hamiltonian and has the property

$$
\hat{P_{s}} Q^{+}\left(s^{\prime}\right) \equiv Q^{+}\left(s^{\prime}\right) P_{s}=\delta_{s s^{\prime}} Q^{+}\left(s^{\prime}\right)
$$

One might attempt to calculate $P_{s}$ by means of an expansion in powers of the perturbation $V$. We would then get a set of perturbation theory equations for the corrections to $P_{s}^{0}$ and the 'excitation energy' would be zero through all orders. Equation (1) does not have a unique solution when $\omega$ equals zero, however, not even when the 
normalisation condition $\left(P_{s}^{0} \mid P_{s}\right)=1$ is invoked. Thus instead, we choose to express the projector $P_{s}$ in terms of the excitation operator $Q^{+}(s)$ as

$$
P_{s}=\frac{Q(s) Q^{+}(s)}{\operatorname{Tr}\left(Q(s) Q^{+}(s)\right)}
$$

If $Q^{+}(s)$ is correct through $n$th order, equations (44) and (45) will hold through this order only.

The transition moment, $M$, for the transition we are studying is now given by

$$
M=\left(P_{s}|R| Q^{+}(s)\right)
$$

where $R$ denotes, for instance, the dipole moment operator. Similarly the total energies of the initial and final state are

$$
E(s)=\left(P_{s} \mid H\right) \quad \text { and } \quad E\left(s^{*}\right)=\left(P_{s}^{*} \mid H\right)
$$

where the projector onto the excited state is

$$
P_{s}^{*}=\frac{Q^{+}(s) Q(s)}{\operatorname{Tr}\left(Q^{+}(s) Q(s)\right)}
$$

Thus it is apparent that the excitation operator $Q^{+}(s)$ contains all information about both the initial and the final state of the transition.

\section{Removal or addition of an electron}

The general manifold of zeroth-order operators $Q_{0}^{+}(s)$ can, according to equation (30), be decomposed into orthonormal and non-interacting subsets of eigenoperators of the superoperator $\hat{N}$, defined as $\hat{N} x=\Sigma_{r}\left[n_{r}, x\right]$ for any operator $x$, according to their eigenvalues. In this section, we apply the perturbative developments of $\S 2$ to those operators $Q_{0}^{+}(s)$ which obey $\hat{N} Q_{0}^{+}(s)= \pm 1 Q_{0}^{+}(s)$, i.e. those which involve addition or removal of a single electron from the parent system whose zeroth-order occupation numbers are $f_{r}(s)$.

The unperturbed electronic Hamiltonian and the perturbation are written as

$$
H_{0}=\sum_{r} \epsilon_{r} n_{r}
$$

and

$$
V=\frac{1}{2} \sum_{r l r^{\prime} l^{\prime}}\left(r l \mid r^{\prime} l^{\prime}\right) a_{r}^{+} a_{r^{\prime}}^{+} a_{l^{\prime}} a_{l}-\sum_{r l} v_{r l} a_{r}^{+} a_{l}
$$

where the Mulliken notation is employed for the two-electron integrals. In equation (48), the orbital energies $\epsilon_{r}$ and the corresponding orbitals $\phi_{r}$ are assumed to have been obtained from a one-electron Schrödinger equation in which the effective potential $v$ appears. This potential may be the Hartree-Fock potential corresponding to occupation numbers $f_{k}(s)$ :

$$
v_{r l}=\sum_{k}(r l \| k k) f_{k}(s)
$$

where $\left(r l \mid r^{\prime} l^{\prime}\right)=\left(r l \mid r^{\prime} l^{\prime}\right)-\left(r l^{\prime} \mid r^{\prime} l\right)$, or it may be any of several other common one-electron potentials. For example, $v$ could be a local exchange potential (Slater 1975), 
one of the so-called $V^{N-1}$ potentials (Huzinaga et al 1973), a pseudopotential (Melius et al 1974), or one of the several varieties of Hartree-Fock potentials.

With the above decomposition of the electronic Hamiltonian, and with the particular zeroth-order ionisation operator $Q_{0}^{+}(s)=a_{p}^{+} P_{s}^{0}$ as our chosen reference, we obtain the following first-order correction (equation (14)) to the Koopmans' theorem electron affinity $\left(\omega_{0}(s)=\epsilon_{p}\right)$ :

$$
\omega_{1}(s)=\sum_{k}(p p \| k k) f_{k}(s)-v_{p p}
$$

The techniques used to evaluate $\omega_{1}(s)$ are illustrated in the appendix. Clearly, if $v$ is equal to the Hartree-Fock potential corresponding to the orbital occupation numbers $f_{k}(s), \omega_{1}(s)$ will vanish. However, the other first-order correction, $\omega_{1}\left(s^{\prime}\right)$, will not be zero, and, in general, $\omega_{1}\left(s^{\prime}\right) \neq \omega_{1}\left(s^{\prime \prime}\right)$ if $s^{\prime} \neq s^{\prime \prime}$, i.e. the degeneracy of the zerothorder operators is lifted in first order.

The first-order correction to the ionisation operator, which is needed to compute $\omega_{2}(s)$ and $\omega_{3}(s)$, is from equation (20)

$$
Q_{1}^{+}(s)=-\frac{\mathrm{i}}{2} \int_{-\infty}^{\infty} \mathrm{d} t \operatorname{sgn}(t) \exp \left[\mathrm{i}\left(\omega_{0}-H_{0}\right) t\right]\left[V, a_{p}^{+} P_{s}^{0}\right] \exp \left(\mathrm{i} H_{0} t\right)
$$

which can be written more explicitly as

$$
\begin{aligned}
& Q_{1}^{+}(s)=\left(-\frac{1}{2} \sum_{r r^{\prime} l^{\prime}}^{*}\left(r p \pi r^{\prime} l^{\prime}\right) a_{r}^{+} a_{r^{\prime}}^{+} a_{l^{\prime}}-\sum_{r} \Delta \tilde{v}_{r p} a_{r}^{+}\right) P_{s}^{0} \\
& \quad-\frac{1}{4} \sum_{r r^{\prime} l l^{\prime}}^{*}\left(r l \pi r^{\prime} l^{\prime}\right) a_{p}^{+}\left[a_{r}^{+} a_{r^{\prime}}^{+} a_{l^{\prime}} a_{l}, P_{s}^{0}\right]-\sum_{r l} \Delta \tilde{v}_{r l} a_{p}^{+}\left[a_{r}^{+} a_{l}, P_{s}^{0}\right]
\end{aligned}
$$

where the * indicates that neither $r$ nor $r^{\prime}$ equals $l^{\prime}$ in the first sum and that neither $r$ nor $r^{\prime}$ equals $l$ or $l^{\prime}$ in the other sum. For notational ease we define

$$
\begin{aligned}
& \Delta v_{r l} \equiv \sum_{k}(r l \| k k) f_{k}(s)-v_{r l} \\
& \Delta \tilde{v}_{r l} \equiv \Delta v_{r l} /\left(\epsilon_{r}-\epsilon_{l}\right) \\
& \left(r l \tilde{l}^{\prime} r^{\prime} l^{\prime}\right) \equiv\left(r l \| r^{\prime} l^{\prime}\right) /\left(\epsilon_{r}+\epsilon_{r^{\prime}}-\epsilon_{l^{\prime}}-\epsilon_{l}\right)
\end{aligned}
$$

and the quantities $\Delta \tilde{v}_{r l}$ and $\left(r l \tilde{\|} r^{\prime} l^{\prime}\right)$ are defined to be zero whenever the denominator vanishes. To obtain the second-order correction to the ionisation energy we compute

$$
\omega_{2}(s)=\left(Q_{0}^{+}(s)|\hat{V}| Q_{1}^{+}(s)\right)
$$

for which we find

$$
\begin{aligned}
& \omega_{2}(s)=\sum_{\substack{m<n \\
\alpha}}(p m \pi \alpha n)(n \alpha \pi m p)+\sum_{\substack{\alpha<\beta \\
m}}(p \alpha \pi m \beta)(\beta m \| \alpha p) \\
& \quad+\sum_{m, \alpha}\left[(p p \pi \alpha m) \Delta v_{m \alpha}+\Delta v_{\alpha m}(m \alpha \pi p p)\right]+\sum_{l} \Delta \tilde{v}_{p l} \Delta v_{l p}
\end{aligned}
$$

where the orbitals $m, n(\alpha, \beta)$ have occupation number equal to zero (unity) in the list $\left\{f_{r}(s)\right\}$.

If $v$ is chosen equal to the Hartree-Fock potential $\left(v_{\mathrm{HF}}(s)\right)$ corresponding to occupancies $\left\{f_{r}(s)\right\}, \Delta v=0$ and $\omega_{2}(s)$ is equal to the standard second-order RayleighSchrödinger perturbation theory estimate of the ion-neutral energy difference (Pickup 
and Goscinski 1973). In this case, $\omega_{2}(s)$ is also identical to a diagonal element of the second-order part of the self-energy or optical potential evaluated at $E=\omega_{0}(s)$

$$
\omega_{2}(s)=\Sigma_{p, p}^{(2)}\left(E=\epsilon_{p}\right) \text {. }
$$

For this specific choice of $v$, the other $\omega_{2}\left(s^{\prime}\right)\left(s^{\prime} \neq s\right)$ must still be calculated from equation (58). The more general result given in equation (58) also allows one to compute ionisation energies which are correct through second order for other choices of the potential $v$ such as those mentioned earlier in this section. Equivalent results were derived diagrammatically by Paldus and Cizek (1975).

To obtain the third-order correction to the above ionisation energy, we must calculate

$$
\omega_{3}(s)=\left(Q_{0}^{+}(s)|\hat{V}| Q_{2}^{+}(s)\right)
$$

which by using equations (5) and (6) can be rewritten in terms of $Q_{1}^{+}(s)$ as

$$
\omega_{3}(s)=\left(Q_{1}^{+}(s)\left|\hat{V}-\omega_{1}(s)\right| Q_{1}^{+}(s)\right) .
$$

Because we do not have to solve for $Q_{2}^{+}(s)$, and because $\kappa_{1}\left(s^{\prime \prime}\right)=0$, we need not employ either of the two previously discussed treatments of the degeneracy of $\omega_{0}(s)$. This problem would first arise in calculating $\omega_{4}(s)$.

In the case for which the reference potential $v$ is chosen to be equal to $v_{\mathrm{HF}}(s)$, we obtain:

$$
\begin{aligned}
& \omega_{3}(s)=+\sum_{\substack{r<q \\
\alpha, \beta, \gamma}}(p p \| \beta \gamma)(r \alpha \tilde{\|} q \beta)(\alpha r \tilde{\pi} \gamma q) \\
& +\sum_{\substack{r, q \\
\alpha, \beta, \gamma}}(p \beta \pi r \gamma)(\gamma \alpha \| q r)(\alpha p \pi \beta q) \\
& +\sum_{\substack{\delta>\gamma \\
\alpha, \beta, r}}(p \gamma \pi r \delta)(\gamma \alpha \| \delta \beta)(\alpha p \pi \beta r) \\
& +\sum_{\substack{r, q \\
\alpha, \beta, \gamma}}(p \alpha \pi r \beta)(\alpha r \pi \gamma q)(q p \| \beta \gamma) \\
& +\sum_{\substack{r, q \\
\alpha, \beta, \gamma}}(p r \| \gamma \beta)(q \alpha \pi r \gamma)(\alpha p \pi \beta q) \\
& -\sum_{\substack{\alpha, \beta \\
m, r, q}}(p p \pi \alpha m)(m r \| \beta q)(r \alpha \pi q \beta) \\
& +\frac{1}{2} \sum_{\substack{x, \beta \\
m, r, q}}(p \alpha \pi m \beta)(\alpha r \pi \beta q)(r p \| q m) \\
& +\frac{1}{2} \sum_{\substack{\alpha<\beta \\
m, r, q}}(p r \| m q)(r \alpha \pi q \beta)(\alpha p \pi \beta m) \\
& +\mathrm{I}^{\prime}+\mathrm{II}^{\prime}+\mathrm{III}^{\prime}-\mathrm{IV}^{\prime}-\mathrm{V}^{\prime}-\mathrm{VI}^{\prime}-\mathrm{VII}^{\prime}-\mathrm{VIII}^{\prime}
\end{aligned}
$$


where each primed term is formed from the respective unprimed term by performing the substitutions: $\alpha \rightarrow m, \beta \rightarrow n, \gamma \rightarrow r, \delta \rightarrow q, r \rightarrow \alpha, m \rightarrow \gamma$ and taking the complex conjugate.

The analogous expression giving $\omega_{1}(s), \omega_{2}(s)$ and $\omega_{3}(s)$ for the choice of zerothorder operator which involves removal of an electron from spin orbital $\phi_{h}\left(Q_{0}^{+}(s)=a_{h} P_{s}^{0}\right)$ can be obtained from the above results by simply replacing $\phi_{p}$ by $\phi_{h}, \epsilon_{p}$ by $\epsilon_{h}$ and changing the sign. Thus, the perturbative solution of the operator equation of motion is, as expected, equally suited to treatment of electron affinities and ionisation potentials. Results equivalent to equation (62) were given by Cederbaum (1973) in the form of a diagrammatic expansion of the electron propagator self-energy.

\section{Particle-hole excitations}

A very important type of excitation in atomic or molecular systems, which is observed in ultraviolet spectroscopy, may be classified as being of particle-hole type. The zeroorder excitation operator and energy are then

$$
Q_{0}^{+}(s)=a_{p}^{+} a_{h} P_{s}^{0}
$$

and

$$
\omega_{0}(s)=\epsilon_{p}-\epsilon_{h}
$$

respectively. The first-order correction to the excitation energy is obtained from equations (22) and (49) as

$$
\omega_{1}(s)=\operatorname{Tr}\left(P_{s}^{0}\left[a_{h}^{+} a_{p},\left[V, a_{p}^{+} a_{h}\right]\right)=-\left(p p^{\|} h h\right)+\Delta v_{p p}-\Delta v_{h h} .\right.
$$

When the potential $v$ is the Hartree-Fock potential given by equation (50) this result reduces to $-(p p \| h h)$, a well known result in propagator theory (Linderberg and Öhrn 1973). The degeneracy of the unperturbed operators $\left\{Q_{0}^{+}\left(s^{\prime \prime}\right)\right\}$ is again lifted in first order since the difference

$$
\omega_{1}(s)-\omega_{1}\left(s^{\prime}\right)=\sum_{r}[(p p \| r r)-(h h \| r r)]\left(f_{r}(s)-f_{r}\left(s^{\prime}\right)\right)
$$

will be non-vanishing for a general set of spin orbitals except when $f_{r}(s)=f_{r}\left(s^{\prime}\right)$ for all $r \neq h, p$, i.e. when $s=s^{\prime}$, since $f_{h}(s)=f_{h}\left(s^{\prime}\right)=1$ and $f_{p}(s)=f_{p}\left(s^{\prime}\right)=0$. It should be noted that we assume that no orbital energies or two-electron integrals are equal by symmetry. Degeneracies related to the possible angular momentum symmetry, spin symmetry or point-group symmetry of the Hamiltonian may be treated as usual by choosing the proper tensor components for the unperturbed excitation operators. For simplicity we do not consider this problem here.

The calculation of the first-order correction to the excitation operator is similar to the calculation in the preceding section. We write the results as

$$
\begin{aligned}
Q_{1}^{+}(s)=\sum_{r}\left[(r p \pi h h)-\Delta \tilde{i}_{r p}\right] a_{r}^{+} a_{h} P_{s}^{0} \\
-\frac{1}{2} \sum^{*}\left(r p \pi r^{\prime} l^{\prime}\right) a_{r}^{+} a_{r^{\prime}}^{+} a_{l^{\prime}} a_{h} P_{s}^{0}
\end{aligned}
$$




$$
\begin{aligned}
& +\sum_{l} \Delta \tilde{v}_{h l} a_{p}^{+} a_{l} P_{s}^{0} \\
& +\frac{1}{2} \sum^{*}\left(h l \pi r^{\prime} l^{\prime}\right) a_{p}^{+} a_{r^{\prime}}^{+} a_{l^{\prime}} a_{l} P_{s}^{0} \\
& -\sum_{r l} \Delta \tilde{v}_{r l} a_{p}^{+} a_{h}\left[a_{r}^{+} a_{l}, P_{s}^{0}\right] \\
& -\frac{1}{4} \sum^{*}\left(r l \pi r^{\prime} l^{\prime}\right) a_{p}^{+} a_{h}\left[a_{r}^{+} a_{r^{\prime}}^{+} a_{l^{\prime}} a_{l}, P_{s}^{0}\right] .
\end{aligned}
$$

The * has the same meaning as in equation (53).

We are now ready to calculate the second-order correction to the excitation energy. First we write

$$
\omega_{2}(s)=\left(Q_{1}^{+}(s)\left|\left(\omega_{0}-\hat{H}_{0}\right)\right| Q_{1}^{+}(s)\right) .
$$

This form is convenient since the only effect of $\left(\omega_{0}-\hat{H}_{0}\right)$ on $Q_{1}^{+}(s)$ is to remove all denominators and change the sign of all terms in equation (67). The calculation of $\omega_{2}(s)$ is then equivalent to the evaluation of the norm of $Q_{1}^{+}(s)$, which is facilitated by the orthogonality properties of the operators appearing in equation (67). The overlap matrix for these operators has the structure

$\begin{array}{lllllll} & \text { I } & \text { II } & \text { III } & \text { IV } & \text { V } & \text { VI } \\ \text { I } & \text { X } & 0 & 0 & 0 & 0 & 0 \\ \text { II } & 0 & \text { X } & \text { X } & \text { X } & \text { X } & 0 \\ \text { III } & 0 & \text { X } & \text { X } & 0 & 0 & 0 \\ \text { IV } & 0 & \text { X } & 0 & \text { X } & \text { X } & 0 \\ \text { V } & 0 & \text { X } & 0 & \text { X } & \text { X } & 0 \\ \text { VI } & 0 & 0 & 0 & 0 & 0 & \text { X }\end{array}$

and we observe that the number of distinct operator scalar products we have to evaluate, in order to find $\omega_{2}(s)$, is 10 . The sum of these terms may be written as

$$
\begin{aligned}
& \omega_{2}(s)=\sum_{n}\left[(h h \pi p n)-\Delta \tilde{v}_{p n}\right]\left[(n p \| h h)-\Delta v_{n p}\right] \\
&+\left.\sum_{\alpha}\left[(p p \| \alpha h)-\Delta \tilde{v}_{\alpha h}\right][h \alpha \| p p)-\Delta v_{h \alpha}\right] \\
&+\sum_{n} \Delta \tilde{v}_{n h} \Delta v_{h n}+\sum_{\alpha} \Delta \tilde{v}_{p \alpha} \Delta v_{\alpha p} \\
&+\sum_{\substack{n \neq p \\
\alpha \neq h}}[(h h \pi n \alpha)(\alpha n \| p p)+(p p \pi n \alpha)(\alpha n \| h h) \\
&+(h p \pi x n)(n \alpha \| p h)+(p h \pi \alpha n)(n \alpha \| h p) \\
&\left.+\Delta v_{x n}(n \alpha \pi h h)+(h h \| \alpha n) \Delta \tilde{v}_{n x}+\Delta \tilde{i}_{x n}(n \alpha \| p p)+(p p \pi \alpha n) \Delta v_{n x}\right] \\
&+\sum_{\substack{n>m \\
\alpha \neq h}}[(n \alpha \pi m h)(h m \| \alpha n)+(\alpha n \pi p m)(m p \| n \alpha)] \\
&+\sum_{\substack{\alpha>\beta \\
n \neq p}}[(n \alpha \pi p \beta)(\beta p \| \alpha n)+(\alpha n \pi \beta h)(h \beta \| n \alpha)] .
\end{aligned}
$$


Again a considerable simplification is obtained when the orbitals are calculated using a Hartree-Fock potential corresponding to the occupation numbers $\left\{f_{k}(s)\right\}$. In this case the second-order excitation energy correction agrees with the result of Oddershede and Jørgensen (1977) derived from a perturbation expansion of the polarisation propagator self-energy. This comparison is of course only a check on the calculations. Paldus and Cizek (1975) have analysed the particle-hole excitation energy diagrammatically and given results equivalent to equation (70).

\section{Concluding remarks}

In this paper we have shown how one can solve operator equations of motion in a perturbative manner. The essential ingredients which allow us to develop a rigorous solution of these perturbation equations, which in the examples considered are degenerate in zeroth order, are the trace form of operator scalar product and the symmetry projectors which remove this degeneracy in first order. This operator scalar product fulfils all the axioms of a scalar product defined on a linear space. The solution of the operator equation of motion through any order is shown to result in a projector which permits the calculation of transition moments and expectation values, which are correct through the same order.

As applications of the theory we studied electronic ionisation and excitation processes. The ionisation energies obtained by solving the operator equations were shown to agree, through third order, with the results of perturbation treatments in Green's function theory, a comparison that serves as a check on the calculations. Explicit formulae were given for electron affinities (and ionisation potentials) and particle-hole excitation energy through third and second order, respectively. We have included through second order, the possibility that a general one-particle potential is used to define the zeroth-order Hamiltonian.

The approach to many-body perturbation theory employed here is similar to the perturbation theory for propagators, which is also conveniently studied using superoperators, operator scalar products, inner projections and partitioning techniques (Goscinski and Lukman 1970, Pickup and Goscinski 1973, Jørgensen and Simons 1975, Nerbrant 1976). The propagator theory is based on a different operator scalar product, however. Both procedures aim at direct calculation of observable quantities such as transition moments and energies. While the propagator has a set of poles in the complex plane corresponding to a certain manifold of transitions, the present application of Rayleigh-Schrödinger perturbation theory for excitation operators concentrates on one particular transition. Our approach is completely equivalent to standard perturbation theory for the individual states involved in the transition studied. The possible advantage of the operator treatment lies in the compact expressions it offers for the quantities of interest.

\section{Appendix}

The purpose of this appendix is to give a brief description of how the operator scalar products are calculated in practice. Using the anticommutation relations for creation and annihilation operators,

$$
\begin{aligned}
& {\left[a_{r}^{+}, a_{s}\right]_{+}=\delta_{r s}} \\
& {\left[a_{r}, a_{s}\right]_{+}=0}
\end{aligned}
$$


any of the scalar products involved in $\$ \S 3$ and 4 of this paper may be written as a sum of terms like

$$
x=\operatorname{Tr}\left(P_{s}^{0} a_{k}^{+} a_{l}^{+} \ldots a_{l^{\prime}} a_{k^{\prime}}\right) .
$$

When the projector, $P_{s}^{0}$, is given by equation (24), the trace will vanish unless all the indices $k l \ldots$ match the indices ... $l^{\prime} k^{\prime}$ so that we may write

$$
x=\left|\begin{array}{ccc}
\delta_{k k^{\prime}} & \delta_{k l^{\prime}} & \cdots \\
\delta_{l k^{\prime}} & \delta_{l l^{\prime}} & \cdots \\
\cdot & \cdot & \\
\cdot & \cdot &
\end{array}\right| \operatorname{Tr}\left(P_{s}^{0} n_{k} n_{l} \cdots\right) .
$$

The trace of a product of commuting factors, which may be looked upon as acting on different spaces. is equal to the product of the traces of the individual factors over the corresponding space, i.e.

$\operatorname{Tr}\left(P_{s}^{0} n_{k} n_{l} \ldots\right)=\prod_{r \neq k, l, \ldots} \operatorname{Tr}^{(r)}\left[\left(1-f_{r}(s)-n_{r}\right)(-1)^{f_{r}(s)}\right] \prod_{r=k, l, \ldots} \operatorname{Tr}^{(r)}\left[\left(-f_{r}(s) n_{r}\right)(-1)^{f_{r}(s)}\right]$

where

$$
\operatorname{Tr}^{(r)}(A)=\langle\operatorname{vac}|A| \mathrm{vac}\rangle+\left\langle\operatorname{vac}\left|a_{r} A a_{r}^{+}\right| \mathrm{vac}\right\rangle .
$$

We find then

$$
\operatorname{Tr}\left(P_{s}^{0} n_{k} n_{l} \ldots\right)=f_{k}(s) f_{l}(s) \ldots
$$

since the occupation numbers are either zero or one, which when used in equation (A.3) completes the evaluation of $x$.

\section{References}

Banwell C N and Primas H 1963 Molec. Phys. 6225

Cederbaum L S 1973 Theor. Chim. Acta 31239

Condon E V and Shortley G H 1935 The Theory of Atomic Spectra (London: Cambridge University Press)

Crawford J A 1958 Nuovo Cim. 10698

Dirac P A M 1966 Lectures on Quantum Field Theory (London: Academic Press)

Goscinski O and Lukman B 1970 Chem. Phys. Lett. 7573

Huzinaga S, McWilliams D and Cantu A A 1973 Adv. Quantum Chem. 7187

Jørgensen P and Simons J 1975 J. Chem. Phys. 635302

Linderberg J and Öhrn Y 1973 Propagators in Quantum Chemistry (London and New York: Academic Press)

Löwdin P O 1968 Int. J. Quantum Chem. 2867 (and references herein)

1976 Set Theory and Linear Algebra Uppsala TN472 unpublished lecture notes p 3.4.45

Melius C F, O'Lafson B D and Goddard W A 1974 Chem. Phys. Lett. 28459

Nerbrant P O 1976 Int. J. Quantum Chem. 10233

Oddershede J and Jorgensen P 1977 Chem. Phy's. Lett. 4761

Paldus J and Cizek J 1975 Adv, Quantum Chem. 9105

Pickup B T and Goscinski O 1973 Molec. Phys, 261013

Primas H 1961 Helv. Phys, Acta 34331

Simons J 1977 Int. J. Quantum Chem. to be published

Slater J C 1975 Int. J. Quantum Chem. Symp. 97 